\title{
Rare Presentation of Ceftriaxone-Induced Hypersensitivity Pneumonitis
}

\author{
Bushra Komala, b, Stephen Catalya ${ }^{a}$, Jamal Ansaria, Joseph Heliniskia, \\ Shuvendu Sen ${ }^{\text {a }}$, Mayer Ezer ${ }^{\mathrm{a}}$
}

\begin{abstract}
Hypersensitivity pneumonitis (HP), also called extrinsic allergic alveolitis, is a complex syndrome of varying intensity, clinical presentation and natural history rather than a single, uniform disease. Numerous inciting agents have been described including, but not limited to, agricultural dusts, bioaerosols, microorganisms (fungal, bacterial, or protozoal), and certain drugs. Prompt diagnosis of HP is important, as the disease is reversible when diagnosed early in its course. Correct diagnosis is based upon exposure history, clinical assessment, radiographic and physiologic findings, and, if possible, the response to avoidance of the suspected etiologic agent.
\end{abstract}

Keywords: Ceftriaxone; Hypersensitivity pneumonitis; Drug-induced pneumonitis

\section{Introduction}

Drug-induced hypersensitivity pneumonitis is an immune response, normally associated with inhaled agents that can present with varying degrees of respiratory distress. It is important to recognize the effect of drug reaction on the body and differentiate from primary lung pathology. We present a case of hypersensitivity pneumonitis induced by a commonly administered medication, ceftriaxone [1]. This patient originally presented asymptomatic, with a rapid progression and then resolution of symptoms.

\section{Case Report}

This is a 60-year-old male, receiving treatment for right hallux osteomyelitis with ceftriaxone and vancomycin as an outpatient. On a routine follow-up visit, he was sent to the hospital after presenting with a fever, as well as an incidental finding

Manuscript submitted March 9, 2018, accepted April 17, 2018

aRaritan Bay Medical Centre, Perth Amboy, NJ 08861, USA

${ }^{\mathrm{b}}$ Corresponding Author: Bushra Komal, Raritan Bay Medical Centre, Perth Amboy, NJ 08861, USA. Email: komali5@hotmail.com

doi: https://doi.org/10.14740/jmc3046e of elevated creatinine. Upon admission, only vancomycin was discontinued, and admission chest X-ray (CXR) was within normal limits (Fig. 1). On admission day two, he developed acute respiratory failure requiring mechanical ventilation with CXR findings of right-sided infiltrate and non-specific leftsided changes (Fig. 2). No skin rash was noted and the labs did not show eosinophilia at the time. Bronchoalveolar lavage was negative for microorganisms, ruling out the differential of $\mathrm{Aci}$ netobacter pneumonia. Ceftriaxone was empirically stopped, given the rare possibility of hypersensitivity pneumonitis. After cessation of ceftriaxone, the patient's condition improved significantly and was extubated, CXR demonstrated improvement (Fig. 3).

\section{Discussion}

The original differential diagnosis for this case was broad, ranging from health-care-associated pneumonia to pulmonary embolism to drug-induced lung injury. The acute presentation, however, helped limit the differential, with interstitial pneumonitis being high on the list. Possible etiologies were ruled out by negative extremities ultrasound and bronchoalveolar

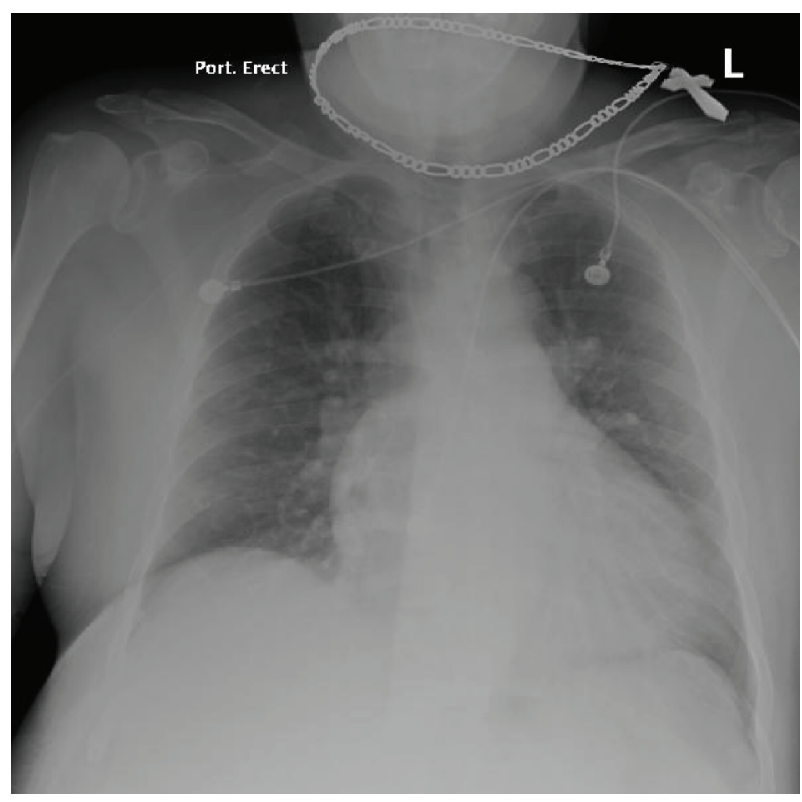

Figure 1. Chest X-ray at admission was "within normal limits". 


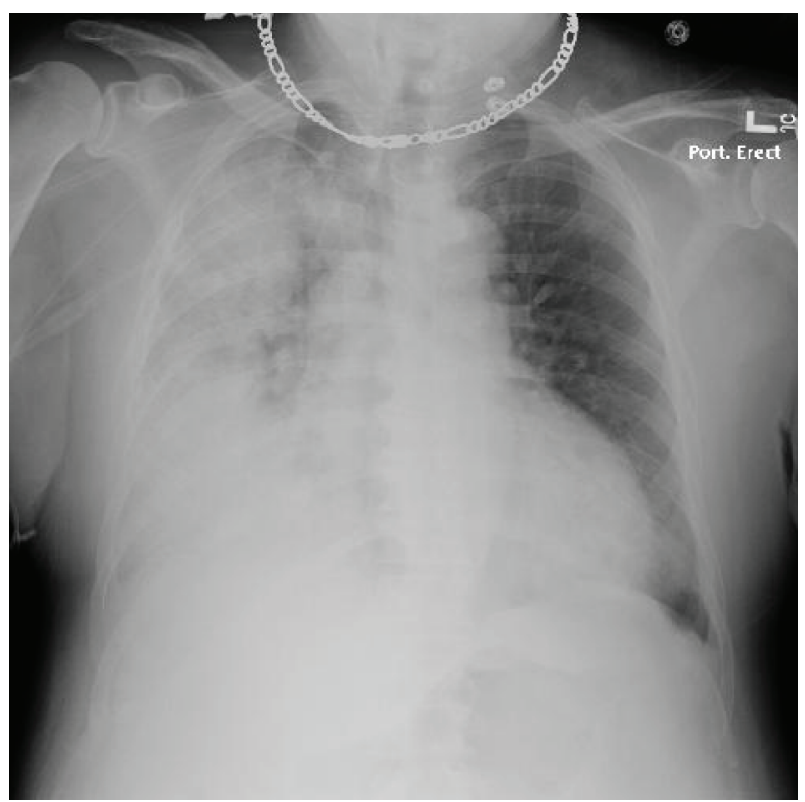

Figure 2. Chest X-ray on admission day two, for evaluation of respiratory distress showing right-sided infiltrate and non-specific left sided changes.

lavage negative for microorganisms. In this case, lack of rash and eosinophilia lessened the possibility of a hypersensitivity reaction, though rapid improvement after cessation of the causative agent was supportive of this diagnosis.

Interstitial pneumonitis is defined as an inflammation change of the lung interstitium, including the alveolar septa, due to a broad range of etiologies. The etiology of interstitial pneumonitis can either be idiopathic or from known causes, such as drug-induced [2, 3]. In immunocompromised populations, interstitial pneumonitis is more commonly caused by fungal, atypical bacterial, and viral [4] infections [5]. In healthy populations, the cause of interstitial pneumonitis is more commonly an extrinsic particulate; fungal or plant antigens in farmer's lung [6], aerosolized metallic or organic chemicals [7], or even possibly radiation [8].

The presentation of drug-induced pneumonitis commonly includes dyspnea, fever, cough, and rash. Investigation will often reveal inflammatory changes on CXR or CT [9], and eosinophilia on lab analysis. If a drug hypersensitivity reaction is suspected as the cause of pneumonitis, the medication can be restarted after cessation to confirm the reappearance of symptoms; this however may cause the patient to require intubation and supportive treatment. As with other hypersensitivity reactions, the first step in treatment of drug-induced pneumonitis, after life supporting measures, is cessation of the drug or inhalation agent [10]. Patients with severe disease may require treatment with corticosteroids [10], however, this has been shown to have no effect on long-term outcomes [11].

\section{Conclusions}

While hypersensitivity reactions are a known adverse effect

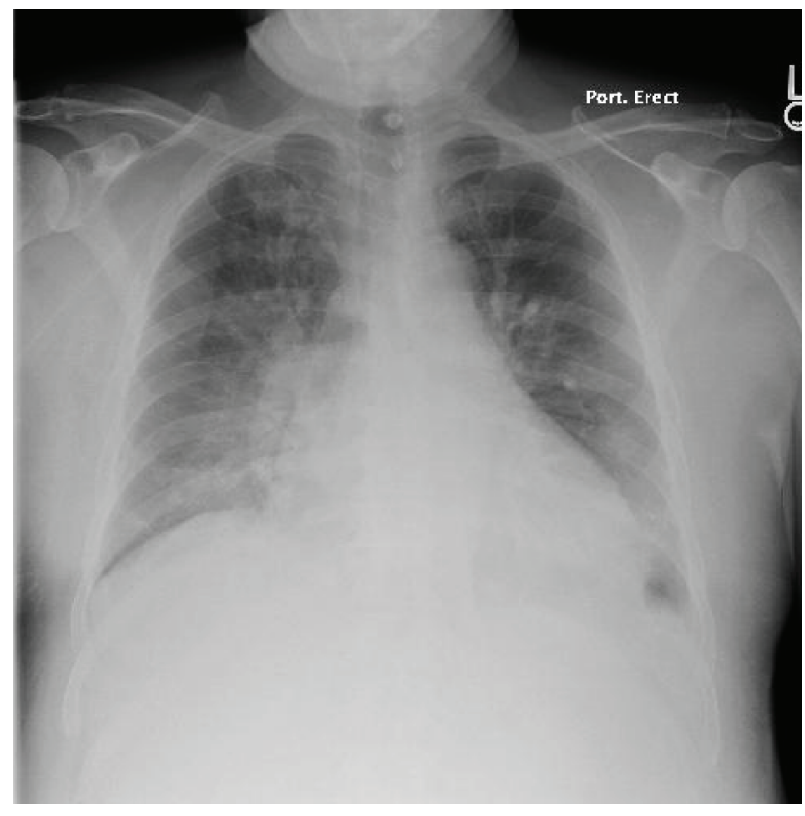

Figure 3. Chest $\mathrm{X}$-ray post ceftriaxone cessation showing resolving right-sided infiltrate.

of the use of cephalosporins, there are few reported cases of pneumonitis associated with ceftriaxone [1]. This case is suggestive that clinicians should continue to be aware of the possibility of drug-induced pneumonitis, even in the absence of rash or eosinophilia. At this time, more investigation is required to establish the exact mechanism behind ceftriaxone-induced pneumonitis, and to determine if there is population with an elevated risk of this complication. We believe that the addition of hypersensitivity pneumonitis to the differentials of patients with similar presentations, and early recognition of this complication can reduce or even prevent significant morbidity and mortality.

\section{References}

1. Lee SH, Kim MH, Lee K, Jo EJ, Park HK. Hypersensitivity pneumonitis caused by cephalosporins with identical R1 side chains. Allergy Asthma Immunol Res. 2015;7(5):518-522.

2. King TE. Epidemiology and causes of hypersensitivity pneumonitis. In: UpToDate, Post TW (Ed), UpToDate, Waltham, MA. (Accessed on December 16, 2016.)

3. King TE. Diagnosis of hypersensitivity pneumonitis. In: UpToDate, Post TW (Ed), UpToDate, Waltham, MA. (Accessed on December 15, 2016.).

4. Travis WD, Fox CH, Devaney KO, Weiss LM, O'Leary TJ, Ognibene FP, Suffredini AF, et al. Lymphoid pneumonitis in 50 adult patients infected with the human immunodeficiency virus: lymphocytic interstitial pneumonitis versus nonspecific interstitial pneumonitis. Hum Pathol. 1992;23(5):529-541.

5. de Blic J, McKelvie P, Le Bourgeois M, Blanche S, Benoist MR, Scheinmann P. Value of bronchoalveolar lav- 
age in the management of severe acute pneumonia and interstitial pneumonitis in the immunocompromised child. Thorax. 1987;42(10):759-765.

6. Malmberg P, Rask-Andersen A, Rosenhall L. Exposure to microorganisms associated with allergic alveolitis and febrile reactions to mold dust in farmers. Chest. 1993;103(4):1202-1209.

7. Robertson W, Robertson AS, Burge CB, Moore VC, Jaakkola MS, Dawkins PA, Burd M, et al. Clinical investigation of an outbreak of alveolitis and asthma in a car engine manufacturing plant. Thorax. 2007;62(11):981-990.

8. Vogelius IR, Bentzen SM. A literature-based meta-anal- ysis of clinical risk factors for development of radiation induced pneumonitis. Acta Oncol. 2012;51(8):975-983.

9. Akira M, Ishikawa H, Yamamoto S. Drug-induced pneumonitis: thin-section CT findings in 60 patients. Radiology. 2002;224(3):852-860.

10. Sostman HD, Matthay RA, Putman CE, Smith GJ. Methotrexate-induced pneumonitis. Medicine (Baltimore). 1976;55(5):371-388

11. Kokkarinen JI, Tukiainen HO, Terho EO. Effect of corticosteroid treatment on the recovery of pulmonary function in farmer's lung. Am Rev Respir Dis. 1992;145(1):35 . 